\title{
Enhancing verbal episodic memory in older and young subjects after non-invasive brain stimulation
}

\author{
Rosa Manenti ${ }^{1}$, Michela Brambilla ${ }^{1,2}$, Michela Petesi ${ }^{1}$, Clarissa Ferrari ${ }^{1}$ and Maria Cotelli ${ }^{1}$ * \\ 1/stituto di Ricovero e Cura a Carattere Scientifico Centro San Giovanni di Dio Fatebenefratelli, Brescia, Italy \\ ${ }^{2}$ Center for Cognitive Science, Department of Psychology, University of Turin, Turin, Italy
}

\section{Edited by:}

Hari S. Sharma, Uppsala University, Sweden

\section{Reviewed by:}

Catarina Oliveira, University of

Coimbra, Portugal

Giovanni Tosi, University of Modena and Reggio Emilia, Italy

\section{*Correspondence:}

Maria Cotelli, Istituto di Ricovero e Cura a Carattere Scientifico Centro San Giovanni di Dio Fatebenefratelli, Via Pilastroni 4, 25125 Brescia, Italy e-mail: mcotelli@fatebenefratelli.it
Memory is the capacity to store, maintain, and retrieve events or information from the mind. Difficulties in verbal episodic memory commonly occur in healthy aging. In this paper, we assess the hypothesis that anodal transcranial direct current stimulation (tDCS) applied over the dorsolateral prefrontal cortex (DLPFC) or over the parietal cortex (PARC) could facilitate verbal episodic memory in a group of 32 healthy older adults and in a group of 32 young subjects relative to a sham stimulation using a single-blind randomized controlled design. Each participant underwent two sessions of anodal tDCS (left and right) and one session of sham stimulation. Overall, our results demonstrated that, in young and in older subjects, anodal tDCS applied during the retrieval phase facilitates verbal episodic memory. In particular, we found that tDCS applied over the left and right regions (DLPFC and PARC) induced better performance in young participants; only tDCS applied over the left regions (DLPFC and PARC) increased retrieval in older subjects. These results suggest that anodal tDCS can be a relevant tool to modulate the long-term episodic memory capacities of young and older subjects.

Keywords: tDCS, aging, verbal retrieval, dorsolateral prefrontal cortex, parietal cortex

\section{INTRODUCTION}

Episodic memory is a fundamental form of long-term memory that relies on different processes to encode, consolidate, and retrieve information (Tulving, 1983). Several studies have shown that aging is associated with decline in the encoding and retrieval of episodic information from previously experienced events (Tulving, 1983; Spencer and Raz, 1995; Balota et al., 2000). These reductions in memory performance most likely reflect age-related changes in the brain, which undergoes significant structural and functional modifications during the aging process (Creasey and Rapoport, 1985). These age-related modifications, which are characterized by reduced activity in the networks dedicated to performing a given function, may be due to decreased cell metabolism (Burke and Barnes, 2006). Based on these age-related changes, an amendment to the hemispherical encoding retrieval asymmetry (HERA) theory (Tulving et al., 1994) was proposed for older adults. The HERA model predicts that in younger adults, the left prefrontal cortex (PFC) would specialize in encoding, while the right PFC would be crucial for retrieval. In older adults, the hemispheric asymmetry reduction (HAROLD) model has been proposed based on functional magnetic resonance imaging (fMRI) studies (Cabeza, 2002). Although the activation of the right PFC during retrieval is less pronounced, bilateral involvement of the PFC during both encoding and retrieval has been repeatedly observed in healthy older adults. Furthermore, dorsolateral prefrontal cortex (DLPFC) lateralization seems to be influenced not only by the process (encoding/retrieval) but also by the material used (verbal/non-verbal) and by the task demand (Kapur et al., 1996; Fletcher et al., 1998; Wagner et al., 1998; Poldrack et al., 1999; Golby et al., 2001).
There are numerous studies establishing the crucial role of the DLPFC in episodic memory; neuroimaging studies have demonstrated the involvement of a distributed neural network formed by the DLPFCs, the medial temporal lobes, the parietal cortices (PARCs) and the precuneus (Rugg and Wilding, 2000; Buckner et al., 2001; Fletcher and Henson, 2001; Cabeza and Nyberg, 2003; Cabeza et al., 2003, 2008; Simons and Spiers, 2003; Berryhill, 2012). Interestingly, processing of abstract and concrete words has been reported to involve different prefrontal and parietal areas (Binder et al., 2005; Klostermann et al., 2008).

Transcranial direct current stimulation (tDCS) is a noninvasive brain stimulation technique that induces long-lasting, stimulation-polarity-dependent excitability shifts in the cerebral cortex (Nitsche and Paulus, 2000, 2001; Nitsche et al., 2003a,b, 2008; Dayan et al., 2013). Recently, tDCS has facilitated memory capacity in young subjects (Boggio et al., 2009a; Chi et al., 2010; Penolazzi etal., 2010; Javadi and Cheng, 2012; Javadi and Walsh, 2012; Javadi etal., 2012; Jacobson et al., 2013) and in patients with Alzheimer's disease (Ferrucci et al., 2008; Boggio et al., 2009b, 2011, 2012). In older subjects, some studies reported improvements in learning (Floel et al., 2012; Zimerman et al., 2013) and working memory (Berryhill and Jones, 2012) after non-invasive brain stimulation. However, no studies have explored the effects of aging on verbal episodic memory using tDCS.

The aim of this study is to assess whether anodal tDCS results in an improvement of episodic memory performance in older and young subjects. Therefore, we compared the retrieval of abstract and concrete words in both young and older subjects during the application of either anodal or placebo tDCS over the DLPFCs 
and PARCs. The choice of DLPFCs and PARCs as the main sites of stimulation was based on results of previous studies which showed the involvement of these two areas in episodic memory tasks (Manenti et al., 2010; Berryhill, 2012). Additionally, this work aimed to gather more information about the role of the PARC and DLPFC in episodic memory. Finally, the comparison of retrieval performances in older and young subjects, allows us to investigate the potential functional compensation of age-related changes in hemispherical asymmetry.

\section{MATERIALS AND METHODS PARTICIPANTS}

Thirty-two healthy young volunteers [mean age $=23.72 \pm 3.15$ years; mean education $=15.13 \pm 2.04$ years $(9$ males, 23 females $)$ ] and 32 healthy older individuals [mean age $=67.91 \pm 4.72$ years; mean education $=10.75 \pm 4.63$ years $(15$ males, 17 females $)$ ] took part in the experiment. All of the subjects had normal or corrected-to-normal vision and were native Italian speakers. See Table 1 for demographic details.

Participants reported being free of neurological disorders and had no history of seizures. All participants were informed about the procedures and the possible risks of tDCS, and written informed consent was obtained after a safety screening. The experimental methods got ethical approval from the local Human Ethics Committee (CEIOC - Ethics Committee of the IRCCS Centro San Giovanni di Dio Fatebenefratelli, Brescia, Italy). Prior to being enrolled in the experiment, older subjects completed a Mini Mental State Examination (MMSE; Folstein et al., 1975) and a detailed neuropsychological evaluation to verify the absence of any cognitive deficit. A pathological score in one or more of the tests was an exclusion criterion. The neuropsychological test battery included measures used to assess non-verbal reasoning (Raven's Colored Progressive Matrices), verbal fluency (phonemic and semantic), visuo-spatial capacity (Rey-Osterrieth Complex Figure, Copy), upper-limb apraxia (De Renzi et al., 1980), attention and executive functions (Trail Making Test A and B). In addition, memory was assessed in depth (Story Recall, ReyOsterrieth Complex Figure Recall, Digit Span, Auditory Verbal Learning Test learning and recall). All of the tests were administered and scored according to standard procedures (Lezak et al., 2004). The results of the cognitive assessments are presented in Table 2.

\section{STIMULI}

The experimental procedure was structured in a first encoding phase followed by a retrieval phase. For the encoding phase we selected, 51 abstract and 51 concrete words from the "Corpus e Lessico di Frequenza dell'Italiano Scritto (CoLFIS)" (Laudanna et al., 1995; Bertinetto et al., 2005). For the retrieval phase, we selected an additional pool of 51 abstract and 51 concrete "new" words. Six words (three abstract and three concrete) were assigned to a practice list; the other words were divided into three experimental blocks. Thus, the retrieval word list consisted of the original encoding or "old" words (48 concrete, 48 abstract) and 96 new words (48 concrete, 48 abstract). On average, the words were $6.8( \pm 1.7)$ letters long with $2.9( \pm 0.7)$ syllables. Abstract and concrete words were balanced according to word length and to variables known to influence memory performance, i.e., word frequency and familiarity. There were significant differences between the two word categories with respect to "concreteness" (concrete $=6.3 \pm 0.7$; abstract $=3.9 \pm 0.8 ; p<0.05$ ) and "imageability" (concrete $=5.9 \pm 0.5$; abstract $=3.3 \pm 0.6 ; p<0.05$ ) based on CoLFIS.

\section{PROCEDURE}

\section{Task procedure}

Subjects were seated in a dimly lit room, facing a computer monitor that was placed $60 \mathrm{~cm}$ from the subject. The stimuli were presented using Presentation software (Version 14.9, www.neurobs.com) running on a personal computer with a 17inch screen. Before starting the experiment, subjects completed a practice run that involved encoding and retrieval of three abstract and three concrete words. Both the encoding and the retrieval phases consisted of three separate blocks of 32 (16 concrete and 16 abstract) trials each. The three blocks were matched for familiarity, frequency, concreteness, imageability and word length $(p>0.05)$.

Encoding phase. During the encoding phase, subjects were presented with a word for $2000 \mathrm{~ms}$, followed by an inter-trial interval of $3000 \mathrm{~ms}$. For each trial, subjects were requested to indicate whether a concrete or an abstract word was presented (left index corresponded to concrete words) by pressing one of two buttons of a response box using both hands. During this phase, subjects were also requested to encode the presented words. The encoding phase was followed by a 5-min delay before the retrieval phase began.

Table 1 | Demographic characteristics of young and older individuals grouped according to area of stimulation.

\begin{tabular}{|c|c|c|c|c|c|c|}
\hline & \multicolumn{3}{|c|}{ Young subjects $(n=32)$} & \multicolumn{3}{|c|}{ Older subjects $(n=32)$} \\
\hline Education (years) & $15.1 \pm 2.0$ & $15.2 \pm 2.1$ & ns & $10.0 \pm 4.8$ & $11.5 \pm 4.5$ & $\mathrm{~ns}$ \\
\hline EHI (\%) & $57.6 \pm 61.1$ & $58.2 \pm 61.4$ & $\mathrm{~ns}^{\#}$ & $88.3 \pm 12.8$ & $85.4 \pm 13.3$ & ns \\
\hline
\end{tabular}

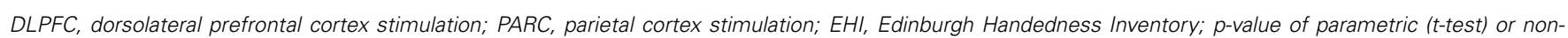
parametric (Mann-Whitney test); ns, not significant.

${ }^{\#}$ p-Value of non-parametric Mann-Whitney test. 
Table 2 | Neuropsychological assessment of older subjects grouped according to area of stimulation.

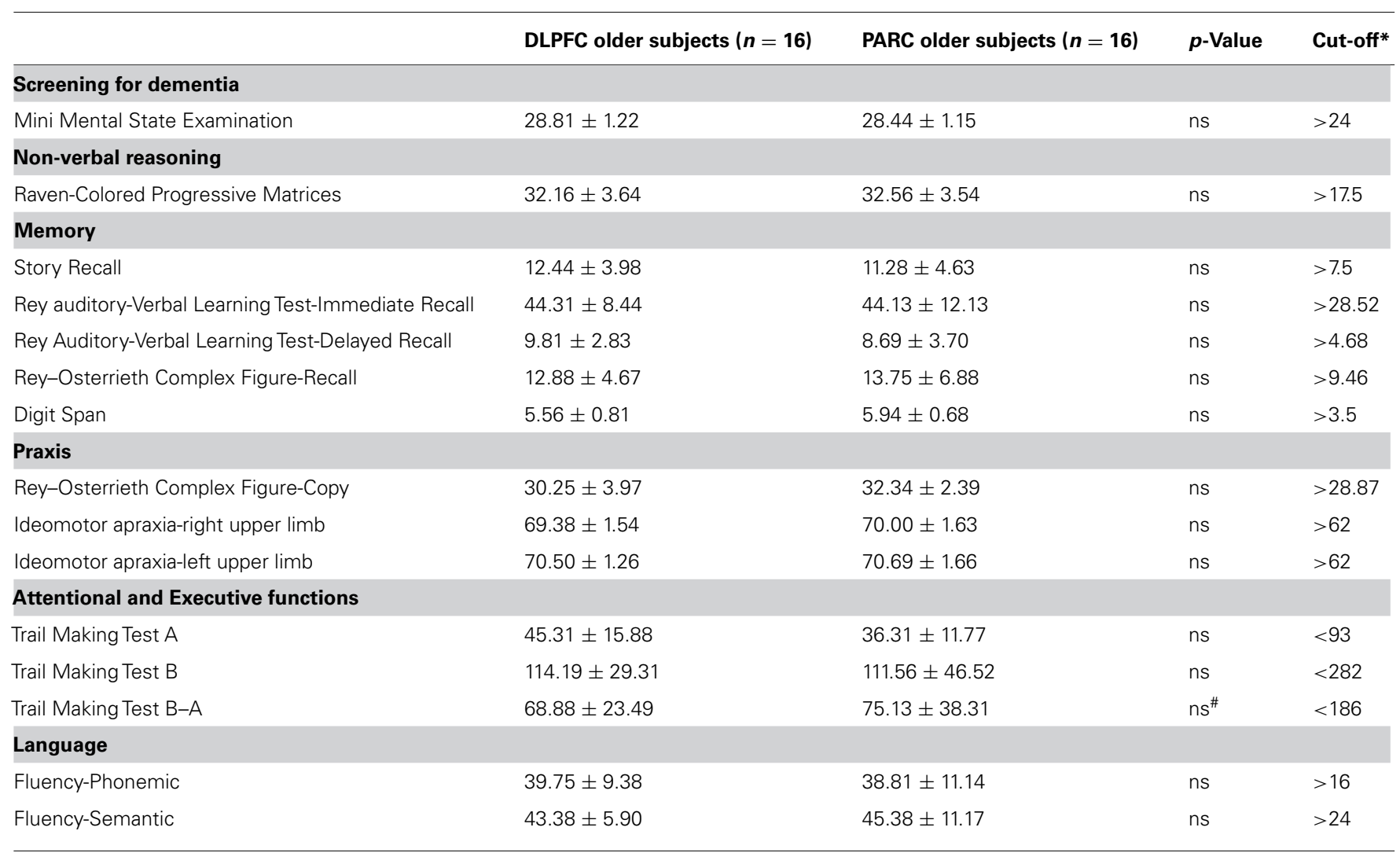

DLPFC, dorsolateral prefrontal cortex stimulation; PARC, parietal cortex stimulation; p-value of parametric (t-test) or non-parametric (Mann-Whitney test); ns, not significant.

${ }^{*}$ Cut-off scores according to Italian normative data are reported. Raw scores are reported; \# $p$-Value of non-parametric Mann-Whitney test.

Retrieval phase. During the retrieval phase, the words presented in the previous encoding block ("old") and the "new" words were displayed in a randomized order. Each word remained on the screen until the subject provided a response. Subjects were instructed to indicate whether the word was "old" or "new" by pressing the right or left button of the button box as soon as possible. For half the subjects, the right button corresponded to "old" choice. Each response was followed by a 2000-ms delay.

In both the encoding and retrieval periods, accuracy and reaction times (RTs) were collected.

The experiment design is illustrated in Figure 1.

\section{tDCS procedure}

The stimulation was delivered by a battery-driven, constant current stimulator (BrainStim, EMS, Bologna, Italy) through a pair of saline-soaked sponge electrodes $(7 \mathrm{~cm} \times 5 \mathrm{~cm})$. A constant current of $1.5 \mathrm{~mA}$ was applied for $6 \mathrm{~min}$ (with a ramping period of $10 \mathrm{~s}$ at the beginning and at the end of the stimulation), starting $2 \mathrm{~min}$ before the beginning of the retrieval task and lasting for the entire retrieval phase. The current density $\left(0.043 \mathrm{~mA} / \mathrm{cm}^{2}\right)$ was maintained below safety limits (Poreisz et al., 2007). The electrodes were secured using elastic bands, and to reduce contact impedance, an electroconductive gel was applied under the electrodes before the montage. In the two age groups, each participant was randomly assigned to either PARC or DLPFC stimulation, yielding two young groups (16 PARC stimulation and 16 DLPFC stimulation) and two older groups (16 PARC stimulation and 16 DLPFC stimulation). The study was a randomized single-blind experiment: the subjects did not know which stimulation they received, but the experimenter did. The three stimuli blocks corresponded to three stimulation conditions: anodal left, anodal right and sham (i.e., placebo).

In the DLPFC groups, the active electrode was placed on the left or right, $8 \mathrm{~cm}$ frontally and $6 \mathrm{~cm}$ laterally with respect to the scalp vertex; in the PARC groups, the active electrode was placed $5 \mathrm{~cm}$ posteriorly and $8 \mathrm{~cm}$ laterally with respect to the scalp vertex. The reference electrode was fixed on the contralateral supraorbital area. In the sham stimulation, the tDCS montage was the same, but the current was turned off $10 \mathrm{~s}$ after the start of the stimulation and was turned on for the last $10 \mathrm{~s}$ of the stimulation period (plus the duration of the fade-in and fade-out periods $=10 \mathrm{~s}$ ),

Therefore, subjects felt the itching sensations below the electrodes at the beginning and at the end of the stimulation, making this condition indistinguishable from the experimental stimulation. Potential tDCS side effects were assessed with a questionnaire (Fertonani etal., 2010) at the end of each session. The active stimulations (i.e., anodal left and anodal right) were executed on two different days to minimize the likelihood of interference 


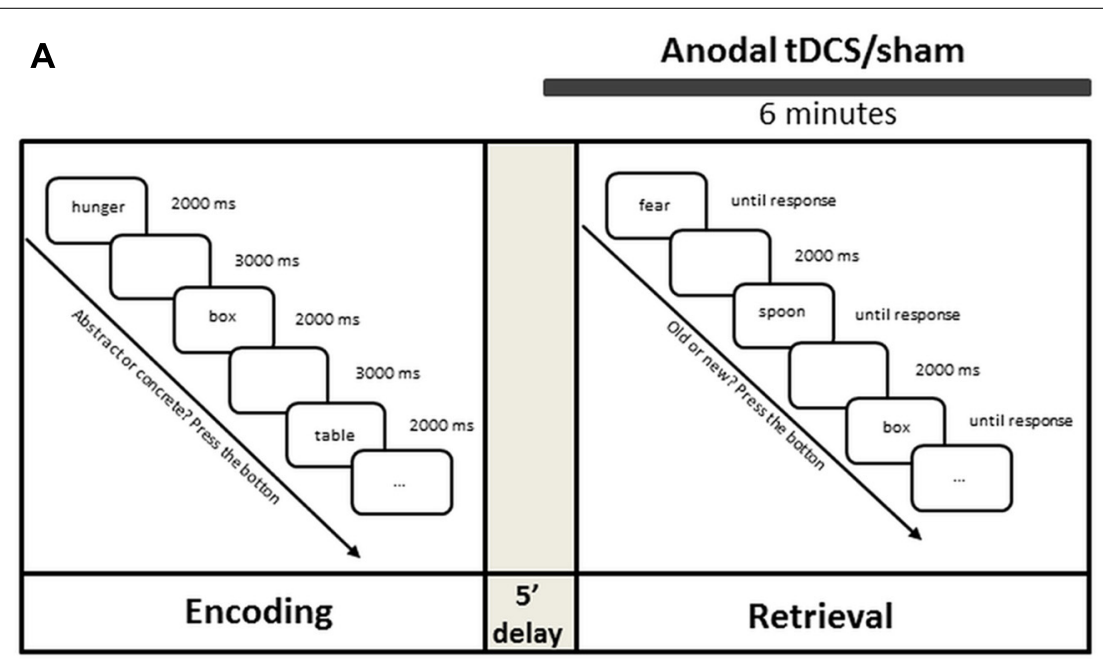

B

\section{Stimulation during Retrieval DLPFC Stimulation}

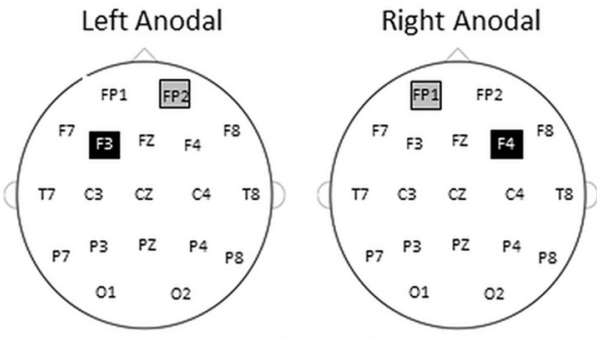

PARC Stimulation Left Anodal

Right Anodal
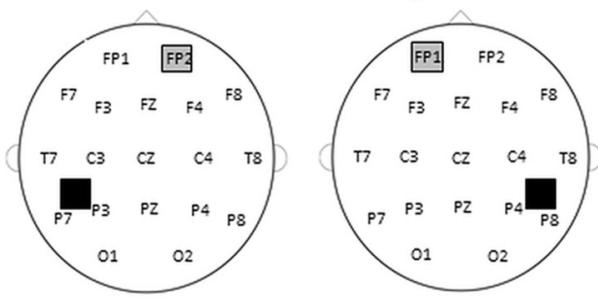

FIGURE 1 | (A) Experimental design. An encoding phase was followed by a retrieval phase. tDCS was applied for 2 min before retrieval and throughout the retrieval. (B) Electrode montage on the dorsolateral prefrontal cortex (DLPFC) and on the parietal cortex (PARC).

effects. The sham stimulation was always performed before the active stimulation. For a schematic representation of the full list of conditions used, see Table 3.

Data analysis. Statistical analyses were performed using Statistica software (version 10; www.statsoft.com) and SPSS (Version 21.0, IBM SPSS Statistics for Windows. Armonk, NY: IBM Corp).

For each age category (young and older), demographic variables (e.g., age and education) were compared between the two stimulation groups (DLPFC and PARC) using parametric ( $t$-test) and non-parametric (Mann-Whitney test) analyses. Moreover, $t$-tests were conducted to compare both subjective sensations induced by the different tDCS protocols and the performance acquired during encoding in the three experimental blocks.

Behavioral data were analyzed for both accuracy and RTs during the retrieval sessions. Accuracy data were analyzed using signal detection theory. For each participant, the $d$ prime $\left(d^{\prime}\right)$ for sensitivity to the previously seen words was estimated (Macmillan and Creelman, 2005). The correct recognition of a previously seen word constituted a hit, while erroneous recognition of a "new" word as an "old" word constituted a false alarm (FA). Hit and FA rates were transformed to $Z$ scores using the standard normalized probability distribution. The $d^{\prime}$ was estimated as the difference between the standardized scores $(Z)$ of the hit rates $(H)$ and of the FA rates. 
Table 3 | Experimental conditions.

\begin{tabular}{lll}
\hline Stimulation site & First session & Second session \\
\hline DLPFC & Sham-anodal right & Anodal left \\
& Sham-anodal left & Anodal right \\
& Anodal right & Sham-anodal left \\
& Anodal left & Sham-anodal right \\
& Sham-anodal right & Anodal left \\
PARC & Sham-anodal left & Anodal right \\
& Anodal right & Sham-anodal left \\
& Anodal left & Sham-anodal right \\
\hline
\end{tabular}

A generalized estimating equations (GEE) model (Hardin and Hilbe, 2003) was adopted to analyze the non-normal (Gamma distributed) dependent variable RT measured according to the experimental design including two within factors: stimulation (left anodal, right anodal and sham), word categories (abstract and concrete); and two between factors: stimulated areas (DLPFC and PARC) and recruited groups (older and young). With the same experimental design, an ANOVA model was performed for the dependent variable accuracy.

Bonferroni corrections were adopted for all comparison adjustments of post hoc analyses.

\section{RESULTS}

No differences in age or education were observed between the stimulation groups in either the young or older subjects.

We also looked for performance differences between blocks during encoding. Block number had no effect on accuracy or RT for either abstract or concrete words, suggesting that the word lists in the three blocks required similar concreteness judgment during encoding.

\section{STIMULATION QUESTIONNAIRE}

Perceptual sensations induced by the anodal tDCS and sham tDCS conditions were assessed with standardized questionnaire developed by Fertonani et al. (2010). Participants were asked to evaluate intensity of several perceptual sensations (i.e. itching, pain, burning, heat, pinching, iron taste, fatigue, effect on performance) through a 5 -point-scale $(0=$ none, $1=$ mild, $2=$ moderate, $3=$ considerable, and $4=$ strong).

By interpreting the questionnaire completed by all subjects at the end of each type of stimulation we inferred that all the subjects well tolerate the stimulation and reported only marginal perceptual sensations. Itch and irritation were the most commonly reported perceptual sensations, with light to moderate intensity. Overall, the experienced perceptual sensations started at the beginning of the experiment, did not last long and did not affect task performance in the anodal or sham conditions. For each group (young and older participants) and each area (DLPFC or PARC), the sensations scores reported during the left and right anodal tDCS were compared with the sensations reported during the sham $\mathrm{tDCS}$ by a single-tailed paired $t$-test. These analyses showed that the anodal stimulations could not be distinguished from the sham stimulation [Young subjects: left DLPFC vs. sham DLPFC, $t=-1.58, \mathrm{df}=15, p=0.14$; right DLPFC vs. sham DLPFC, $t=-0.85, \mathrm{df}=15, p=0.41$; left PARC vs. sham PARC, $t=1.57$, $\mathrm{df}=15, p=0.14$; right PARC vs. sham PARC, $t=1.84$, $\mathrm{df}=15$, $p=0.09$; and Older subjects: left DLPFC vs. sham DLPFC, $t=1.54$, $\mathrm{df}=15, p=0.15$; right DLPFC vs. sham DLPFC, $t=0.24, \mathrm{df}=15$, $p=0.82$; left PARC vs. sham PARC, $t=1.29, \mathrm{df}=15, p=0.22$; right PARC vs. sham PARC, $t=0.25, \mathrm{df}=15, p=0.81]$. There are no reasons to reject the single-blinded character of this study on the basis of these results.

\section{Reaction time analysis}

General $\boldsymbol{t} D C S$ effects. The GEE model that included three types of stimulation (left anodal, right anodal, or sham-placebo, within subjects), two word categories (abstract or concrete, within subjects), two stimulated areas (DLPFC or PARC, between subjects), and two age groups (older or young, between subjects) as factors, revealed significant effects for age (Wald Chi-squared $\chi^{2}=176.15$, $\mathrm{df}=1, p<0.001)$, type of stimulation $\chi^{2}=28.84$, $\mathrm{df}=2$, $p<0.001)$, word category $\left(\chi^{2}=122.72, \mathrm{df}=1, p<0.001\right)$ and the interaction between stimulated areas and the word category $\left(\chi^{2}=4.21, \mathrm{df}=1, p<0.040\right)$. No significant effect was found for the interaction between word category and type of stimulation $\left(\chi^{2}=0.44, \mathrm{df}=2, p<0.801\right)$.

Post hoc analyses (pairwise comparisons of estimated marginal average carried out by Bonferroni adjustment) indicated that older subjects had slower RTs than young individuals (994 ms, 95\% CI [933-1060] vs. $787 \mathrm{~ms}, 95 \%$ CI [751-825], $p<0.001)$; abstract words induced longer RTs than concrete words $(922 \mathrm{~ms}$, 95\% CI [883-962] vs. 849 ms 95\% CI [817-881], $p<0.001)$, and a general facilitation was induced by left tDCS application (left tDCS $=851 \mathrm{~ms}, 95 \%$ CI [819-884] vs. placebo $\mathrm{tDCS}=911 \mathrm{~ms}, 95 \%$ CI [870-954], $p<0.001$; and left tDCS vs. right tDCS $=892 \mathrm{~ms}, 95 \%$ CI [855-930], $p=0.002$ ).

Behavioral effect in young and older subjects. Post hoc pairwise comparisons, evaluated conditionally on sham stimulation, indicated that abstract words induced longer RTs than concrete words in both young (abstract $=847 \mathrm{~ms}$ vs. concrete $=773 \mathrm{~ms}, p<0.001$ ) and older subjects (abstract $=1070 \mathrm{~ms}$ vs. concrete $=985 \mathrm{~ms}$, $p<0.001)$. Moreover, significant differences were found among the two type of words across age categories (for abstract words: young vs. old, $p=0.001$; for concrete words: young vs. old, $p<0.001$ ); see, e.g., Figure 2A.

tDCS effect in young and older subjects. A general facilitation was induced by left tDCS application in older subjects (left $\mathrm{tDCS}=942 \mathrm{~ms}$ vs. placebo $\mathrm{tDCS}=1027 \mathrm{~ms}, p<0.001$; and left $\mathrm{tDCS}$ vs. right $\mathrm{tDCS}=1016 \mathrm{~ms}, p=0.003)$; whereas in young participants, only placebo tDCS $(809 \mathrm{~ms})$ differed from right tDCS (783 ms, $p=0.050)$ and from left tDCS (769 ms, $p=0.026)$. See Figures 3A,B for details.

\section{Accuracy analysis}

General tDCS effects. An ANOVA that included three types of stimulation (left anodal, right anodal, or sham, within subjects), two word categories (abstract or concrete, within subjects), two stimulated areas (DLPFC or PARC, between subjects) and two age groups (older or young, between subjects) as factors showed 


\section{A}

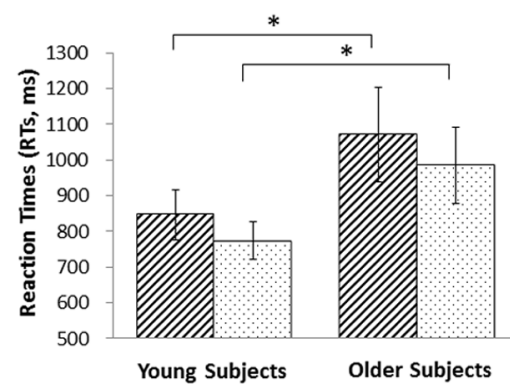

B Accuracy

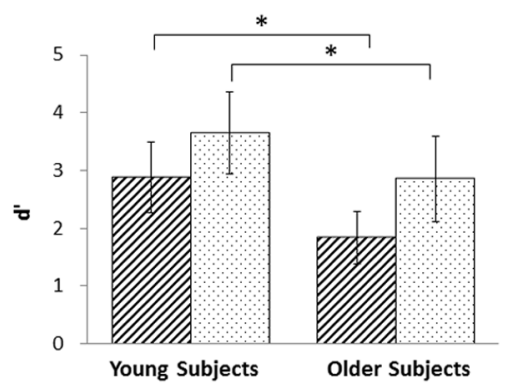

Abstract words

Concrete words

FIGURE 2 | Behavioral effects. (A) Reaction times for abstract and concrete words in the young and older groups. Abstract words induced longer reaction times both in young $(p=0.001)$ and in older $(p=0.001)$ subjects. Moreover, older subjects were slower than young subjects in both abstract $(p=0.0001)$ and concrete words $(p=0.0001)$. (B) Accuracy for abstract and concrete words in the young and older groups. Abstract words induced worse accuracy in both young $(p=0.0001)$ and in older $(p=0.0001)$ subjects. Moreover, older subjects performed worse than young subjects in both abstract $(p=0.0001)$ and concrete words $(p=0.0001)$. Asterisks indicate significant effects $(p<0.05)$.

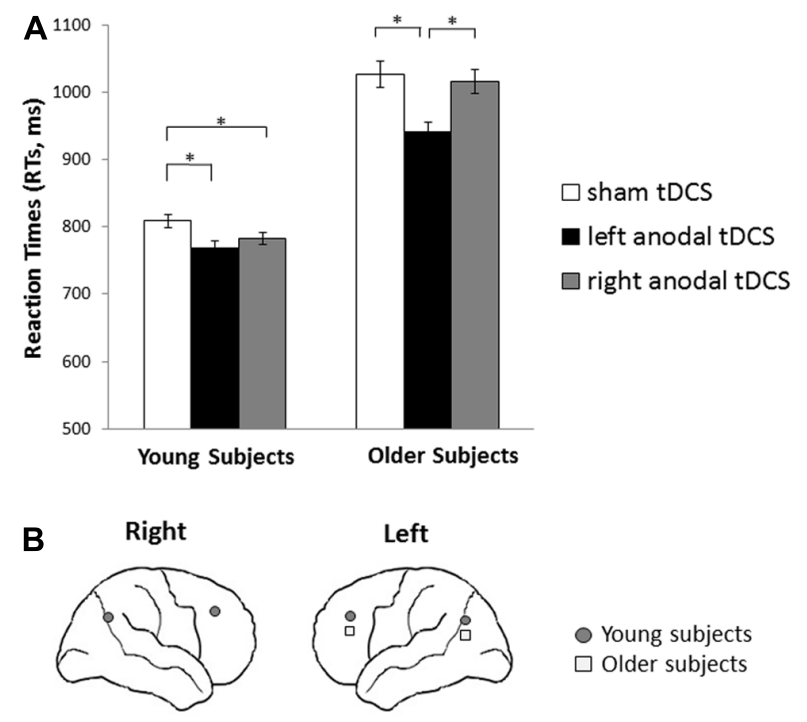

FIGURE 3 | Transcranial direct current stimulation effects on reaction times in young and older subjects during retrieval phase. (A) Reaction times (RTs) achieved during retrieval of words by young and older subjects following tDCS applied over dorsolateral prefrontal cortices (DLPFCs) and parietal cortices (PARCs) compared to sham stimulation. Older participants were consistently faster during left stimulation than during sham stimulation. Young participants were consistently faster during left and right stimulation than during sham stimulation. Asterisks indicate significant effects $(p<0.05)$. (B) Graphical representation of the cerebral areas (DLPFCs and PARCs) related to a reduction of reaction times following anodal tDCS in young and older subjects.

significant effects for age $\left(F_{1,60}=23.764 ; p=0.000008\right)$, word category $\left(F_{1,60}=35.390 ; p=0.000000\right)$, and the interaction between the type of stimulation and the word category $\left(F_{2,120}=4.089\right.$; $p=0.019$ ). Post hoc analyses (Fisher's least significant difference,
LSD) showed that older subjects achieved lower accuracy than young individuals $(2.28 \pm 1.2$ vs. $3.09 \pm 1.3, p=0.000008)$, abstract words induced worse performance than concrete words $(2.31 \pm 1.2$ vs. $3.06 \pm 1.3, p=0.000000)$ and left $t$ DCS application induced a general interference in concrete word recognition (left $\mathrm{tDCS}=2.8 \pm 1.1$, placebo $\mathrm{tDCS}=3.3 \pm 1.5, p=0.000003)$. No other effects were statistically significant. See Figure 2B for details.

\section{DISCUSSION}

Memory is the capacity to store, maintain, and retrieve events or information from the mind. Successful remembering implies a correct encoding and an appropriate retrieval of the information. Overall, our results demonstrated that anodal tDCS applied during the retrieval phase facilitates verbal episodic memory in young and in older subjects. In particular, we found that tDCS applied over the left and right regions (DLPFC and PARC) induces better performance in young participants; only tDCS applied over the left regions (DLPFC and PARC) increased retrieval in older subjects. Remarkably, these facilitation effects were observed during retrieval of both abstract and concrete words.

In agreement with the literature, we found that older subjects experience a significant decline in verbal episodic memory compared to young subjects. Furthermore, our findings suggest that non-invasive brain stimulation, in particular anodal tDCS, applied to left regions could be useful in enhancing memory function in aging. This result agrees with neuroimaging studies that demonstrated an age-related decrease in retrieval that occurred in several regions, including right prefrontal areas and right parietal regions (Grady etal., 1995; Schacter et al., 1996; Cabeza etal., 1997).

Physiological aging induced structural and functional changes have been linked to residual brain plasticity to counteract neural loss (Jancke, 2009). It has therefore been suggested that neural 
plasticity facilitates alternative "strategies" to maintain an adequate level of cognitive performance (Greenwood, 2007; Zollig and Eschen, 2009; Cotelli et al., 2010, 2012).The significance of these changes is intriguing because they could be caused by either an effective functional compensation strategy or an inadequate and/or less efficient processing strategy.

Our data appear to be in line with lesion and functional imaging studies that have indicated that episodic memory involves a widespread network of brain structures, including the PFC and PARCs (Nyberg et al., 2000; Cabeza et al., 2008). Several reviews suggest that both encoding and retrieval are associated with activation in the medial-temporal, prefrontal, and parietal regions. The left ventrolateral PFC and the medial-temporal regions are strongly involved in encoding, whereas the left superior parietal and the dorsolateral and anterior PFC regions are more strongly engaged in retrieval (Spaniol et al., 2009).

Consistent with the HERA model, we observed right involvement during retrieval in young individuals. Moreover, verbal memory retrieval performance was also facilitated when anodal tDCS was applied to left cortical regions (in either young or old individuals). This finding may be consistent with a material specific-model, which postulates that the left hemisphere is engaged in verbal memory processes and the right hemisphere is involved in visuo-spatial memory processes (Wagner et al., 1998; Golby et al., 2001). Furthermore, the asymmetrical left facilitation observed in old subjects was interpreted as reflecting a loss of regional specialization or declining specificity, referred to a dedifferentiation process, which has been hypothesized to occur in physiological aging (Park et al., 2004; Park and Reuter-Lorenz, 2009; Goh et al., 2010).

To investigate the effects of the two different types of encoded material, we compared abstract and concrete word retrieval performance during tDCS. We failed to observe any tDCS difference in abstract and concrete words. In particular, our results revealed that in young and older subjects, both abstract and concrete word retrievals were facilitated by DLPFC and PARC stimulation. The representation of abstract and concrete concepts is an open question in cognitive neuroscience (Kiefer and Pulvermuller, 2012). Neuroimaging studies do not provide converging evidence for neural correlates of abstract and concrete words, suggesting a bilateral representation for concrete words and a less defined network

\section{REFERENCES}

Balota, D. A., Dolan, P. O., and Duchek, J. M. (2000). "Memory changes in healthy young and older adults," in Handbook of Memory, eds E. Tulving and F. I. M. Craik (New York: Oxford University Press), 395-410.

Berryhill, M. E. (2012). Insights from neuropsychology: pinpointing the role of the posterior parietal cortex in episodic and working memory. Front. Integr. Neurosci. 6:31. doi: 10.3389/fnint.2012.00031

Berryhill, M. E., and Jones, K. T. (2012). tDCS selectively improves working memory in older adults with more education. Neurosci.
Lett. 521, 148-151. doi: 10.1016/ j.neulet.2012.05.074

Bertinetto, P. M., Burani, C., Laudanna, A., Marconi, L., Ratti, D., Rolando, C., et al. (2005). Corpus e Lessico di Frequenza dell'Italiano Scritto (CoLFIS). Available at: http://linguistica.sns.it/ CoLFIS/Home.htm

Binder, J. R., Westbury, C. F., McKiernan, K. A., Possing, E. T., and Medler, D. A. (2005). Distinct brain systems for processing concrete and abstract concepts. J. Cogn. Neurosci. 17, 905-917. doi: 10.1162/08989290540 21102

Boggio, P. S., Ferrucci, R., Mameli, F., Martins, D., Martins, O., Vergari, M., et al. (2012). Prolonged visual

(left, right or bilateral) for abstract words (Kiehl et al., 1999; Fiebach and Friederici, 2004; Sabsevitz et al., 2005; Manenti et al., 2010; Rodriguez-Ferreiro et al., 2011). We interpreted the selective involvement of the left areas during the retrieval of abstract and concrete words in older individuals as an expression of a primary use of verbal code and an inefficient mental imagery strategy. This hypothesis is consistent with the idea that the capacity to generate non-verbal mental image strategies declines with age (Johnson and Rybash, 1993; Dror and Kosslyn, 1994; Manenti et al., 2011).

The tDCS technique involves the application of weak electrical currents $(\sim 0.5-2 \mathrm{~mA})$ directly to the head for several minutes ( 5-20 min; Priori, 2003; Nitsche et al., 2008). These currents generate an electrical field that modulates neuronal activity according to the modality of the application. Neurons respond to tDCS by altering their firing rates. Cathodal polarization over the motor cortex can induce reductions in motor cortex excitability, while anodal polarization increases motor cortex excitability (Nitsche and Paulus, 2000). These changes last for minutes to hours beyond the end of the stimulation, depending on the stimulation parameters. Both long-term potentiation and its opposite, long-term depression, have also been postulated to explain the persistent effects of non-invasive brain stimulation on cortical activity (Cooke and Bliss, 2006; Thickbroom, 2007; Ziemann and Siebner, 2008). The long-term effect is a crucial issue for the potential application of these techniques into rehabilitation intervention to ameliorate cognitive deficits.

In conclusion, anodal tDCS can be a relevant tool to modulate the long-term episodic memory capacities of young and older subjects. Memory declines with physiological aging, and memory loss is a characteristic of several clinical conditions. These preliminary findings suggest that anodal tDCS is able to modulate memory performance; this technique could be an interesting approach to study functional adaptation during physiological aging and eventually it could be employed to attempt to reduce the cognitive deficits observed in pathological brain aging.

\section{ACKNOWLEDGMENTS}

This work was supported by a grant from the Alzheimer's Association (NIRG-11-205099). The authors are indebted with Dr Michela Rampini for experimental assistance.

memory enhancement after direct current stimulation in Alzheimer's disease. Brain Stimul. 5, 223-230. doi: 10.1016/j.brs.2011.06.006

Boggio, P. S., Fregni, F., Valasek, C., Ellwood, S., Chi, R., Gallate, J., et al. (2009a). Temporal lobe cortical electrical stimulation during the encoding and retrieval phase reduces false memories. PLoS ONE 4:e4959. doi: 10.1371/journal.pone.0004959

Boggio, P. S., Khoury, L. P., Martins, D. C., Martins, O. E., de Macedo, E. C., and Fregni, F. (2009b). Temporal cortex direct current stimulation enhances performance on a visual recognition memory task in Alzheimer disease. J. Neurol.
Neurosurg. Psychiatry 80, 444-447. doi: 10.1136/jnnp.2007.141853

Boggio, P. S., Valasek, C. A., Campanha, C., Giglio, A. C., Baptista, N. I., Lapenta, O. M., et al. (2011). Non-invasive brain stimulation to assess and modulate neuroplasticity in Alzheimer's disease. Neuropsychol. Rehabil. 21, 703716. doi: 10.1080/09602011.2011. 617943

Buckner, R. L., Wheeler, M. E., and Sheridan, M. A. (2001). Encoding processes during retrieval tasks. J. Cogn. Neurosci. 13, 406-415. doi: $10.1162 / 08989290151137430$

Burke, S. N., and Barnes, C. A. (2006). Neural plasticity in the ageing brain. 
Nat. Rev. Neurosci. 7, 30-40. doi: $10.1038 / \mathrm{nrn} 1809$

Cabeza, R. (2002). Hemispheric asymmetry reduction in older adults: the HAROLD model. Psychol. Aging 17, 85-100. doi: 10.1037/08827974.17.1.85

Cabeza, R., Ciaramelli, E., Olson, I. R., and Moscovitch, M. (2008). The parietal cortex and episodic memory: an attentional account. Nat. Rev. Neurosci. 9, 613-625. doi: 10.1038/nrn2459

Cabeza, R., Grady, C. L., Nyberg, L., McIntosh, A. R., Tulving, E., Kapur, S., et al. (1997). Age-related differences in neural activity during memory encoding and retrieval: a positron emission tomography study. J. Neurosci. 17, 391-400.

Cabeza, R., Locantore, J. K., and Anderson, N. D. (2003). Lateralization of prefrontal activity during episodic memory retrieval: evidence for the production-monitoring hypothesis. J. Cogn. Neurosci. 15, 249-259. doi: 10.1162/089892903321208187

Cabeza, R., and Nyberg, L. (2003) Functional neuroimaging of memory. Neuropsychologia 41, 241244. doi: 10.1016/S0028-3932(02) 00156-2

Chi, R. P., Fregni, F., and Snyder, A. W. (2010). Visual memory improved by non-invasive brain stimulation. Brain Res. 1353, 168-175. doi: 10.1016/j.brainres.2010.07.062

Cooke, S. F., and Bliss, T. V. (2006). Plasticity in the human central nervous system. Brain 129, 1659-1673. doi: 10.1093/brain/awl082

Cotelli, M., Manenti, R., Brambilla, M., Zanetti, O., and Miniussi, C. (2012). Naming ability changes in physiological and pathological aging. Front. Neurosci. 6:120. doi: 10.3389/fnins.2012.00120

Cotelli, M., Manenti, R., Rosini, S., Calabria, M., Brambilla, M., Bisiacchi, P. S., et al. (2010). Action and object naming in physiological aging: an rTMS study. Front. Aging Neurosci. 2:151. doi: 10.3389/fnagi.2010.00151

Creasey, H., and Rapoport, S. I. (1985). The aging human brain. Ann. Neurol. 17, 2-10. doi: 10.1002/ana.410170103

Dayan, E., Censor, N., Buch, E. R., Sandrini, M., and Cohen, L. G. (2013). Noninvasive brain stimulation: from physiology to network dynamics and back. Nat. Neurosci. 16, 838-844. doi: 10.1038/nn.3422

De Renzi, E., Motti, F., and Nichelli, P. (1980). Imitating gestures. A quantitative approach to ideomotor apraxia. Arch. Neurol. 37, 6-10. doi: 10.1001/ archneur.1980.00500500036003
Dror, I. E., and Kosslyn, S. M. (1994). Mental imagery and aging. Psychol. Aging 9, 90-102. doi: 10.1037/08827974.9.1.90

Ferrucci, R., Mameli, F., Guidi, I., Mrakic-Sposta, S., Vergari, M., Marceglia, S., etal. (2008). Transcranial direct current stimulation improves recognition memory in Alzheimer disease. Neurology 71, 493-498. doi: 10.1212/01. wnl.0000317060.43722.a3

Fertonani, A., Rosini, S., Cotelli, M., Rossini, P. M., and Miniussi, C. (2010). Naming facilitation induced by transcranial direct current stimulation. Behav. Brain Res. 208, 311318. doi: 10.1016/j.bbr.2009.10.030

Fiebach, C. J., and Friederici, A. D. (2004). Processing concrete words: fMRI evidence against a specific right-hemisphere involvement. Neuropsychologia 42, 62-70. doi: 10.1016/S0028-3932(03)00145-3

Fletcher, P. C., and Henson, R. N. (2001). Frontal lobes and human memory: insights from functional neuroimaging. Brain 124, 849-881. doi: 10.1093/brain/124.5.849

Fletcher, P. C., Shallice, T., Frith, C. D., Frackowiak, R. S., and Dolan, R. J. (1998). The functional roles of prefrontal cortex in episodic memory. II. Retrieval. Brain 121(Pt 7), 12491256. doi: 10.1093/brain/121.7.1249

Floel, A., Suttorp, W., Kohl, O. Kurten, J., Lohmann, H., Breitenstein, C., et al. (2012). Non-invasive brain stimulation improves objectlocation learning in the elderly. $\mathrm{Neu}$ robiol. Aging 33, 1682-1689. doi: 10. 1016/j.neurobiolaging.2011.05.007

Folstein, M. F., Folstein, S. E., and McHugh, P. R. (1975). "Mini-mental state". A practical method for grading the cognitive state of patients for the clinician. J. Psychiatr. Res. 12, 189-198. doi: 10.1016/00223956(75)90026-6

Goh, J. O., Suzuki, A., and Park, D. C. (2010). Reduced neural selectivity increases fMRI adaptation with age during face discrimination. Neuroimage 51, 336-344. doi: 10.1016/j.neuroimage.2010.01.107

Golby, A. J., Poldrack, R. A., Brewer, J. B., Spencer, D., Desmond, J. E., Aron, A. P., et al. (2001). Materialspecific lateralization in the medial temporal lobe and prefrontal cortex during memory encoding. Brain 124 1841-1854. doi: 10.1093/brain/124. 9.1841

Grady, C. L., McIntosh, A. R., Horwitz, B., Maisog, J. M., Ungerleider, L. G., Mentis, M. J., et al. (1995). Age-related reductions in human recognition memory due to impaired encoding. Science 269, 218 221. doi: 10.1126/science.7618082

Greenwood, P. M. (2007). Functional plasticity in cognitive aging: review and hypothesis. Neuropsychol ogy 21, 657-673. doi: 10.1037/08944105.21.6.657

Hardin, J., and Hilbe, J. (2003). Generalized Estimating Equation. London: CRC Press.

Jacobson, L., Goren, N., Lavidor, M., and Levy, D. A. (2013). Oppositional transcranial direct current stimulation (tDCS) of parietal substrates of attention during encoding modulates episodic memory. Brain Res. 1439, 66-72. doi: 10.1016/j.brainres.2011.12.036

Jancke, L. (2009). The plastic human brain. Restor. Neurol. Neurosci. 27, 521-538. doi: 10.3233/RNN-20090519

Javadi, A. H., and Cheng, P. (2012) Transcranial direct current stimulation (tDCS) enhances reconsolidation of long-term memory. Brain Stimul. 6, 667-674. doi: 10.1016/j.brs.2012.10.007

Javadi, A. H., Cheng, P., and Walsh, V. (2012). Short duration transcranial direct current stimulation (tDCS) modulates verbal memory. Brain Stimul. 5, 468-474. doi: 10.1016/j.brs.2011.08.003

Javadi, A. H., and Walsh, V. (2012). Transcranial direct current stimulation (tDCS) of the left dorsolateral prefrontal cortex modulates declarative memory. Brain Stimul. 5, 231-241. doi: 10.1016/j.brs.2011. 06.007

Johnson, S. H., and Rybash, J. M. (1993). "A cognitive neuroscience perspective on age-related slowing: developmental changes in the functional architecture," in Adult Information Processing: Limits and Loss, eds J. Cerella, J. Rybash, W. Hoyer, and M. L. Commons (New York: Academic Press), 143-173.

Kapur, S., Tulving, E., Cabeza, R., McIntosh, A. R., Houle, S., and Craik, F. I. (1996). The neural correlates of intentional learning of verbal materials: a PET study in humans. Brain Res. Cogn. Brain Res. 4, 243 249. doi: 10.1016/S0926-6410(96) 00058-4

Kiefer, M., and Pulvermuller, F. (2012). Conceptual representations in mind and brain: theoretical developments, current evidence and future directions. Cortex 48 , 805-825. doi: 10.1016/j.cortex.2011. 04.006

Kiehl, K. A., Liddle, P. F., Smith, A. M., Mendrek, A., Forster, B. B., and Hare, R. D. (1999). Neural pathways involved in the processing of concrete and abstract words. Hum. Brain Mapp. 7, 225-233. doi: 10.1002/(SICI) 1097-0193(1999)7:4

Klostermann, E. C., Kane, A. J., and Shimamura, A. P. (2008). Parietal activation during retrieval of abstract and concrete auditory information. Neuroimage 40, 896901. doi: 10.1016/j.neuroimage.2007. 10.068

Laudanna, A., Thornton, A. M., Brown, G., Burani, C., and Marconi, L. (1995). "Un corpus dell'italiano scritto contemporaneo dalla parte del ricevente," in III Giornate Internazionali di Analisi Statistica dei Dati Testuali, Vol. I, eds S. Bolasco, L. Lebart, and A. Salem (Roma: Cisu), 103-109.

Lezak, M., Howieson, D., and Loring, D. W. (2004). Neuropsychological Assessment, 4th Edn. New York: Oxford University Press.

Macmillan, N. A., and Creelman, C. D. (2005). Detection Theory: A User's Guide. Mahwah, NJ: Lawrence Erlbaum Associates.

Manenti, R., Cotelli, M., and Miniussi, C. (2011). Successful physiological aging and episodic memory: a brain stimulation study. Behav. Brain Res. 216, 153-158. doi: 10.1016/j.bbr.2010.07.027

Manenti, R., Tettamanti, M., Cotelli, M., Miniussi, C., and Cappa, S. F. (2010). The neural bases of word encoding and retrieval: a fMRI-guided transcranial magnetic stimulation study. Brain Topogr. 22, 318-332. doi: 10.1007/s10548-009-0126-1

Nitsche, M. A., Cohen, L. G., Wassermann, E. M., Priori, A., Lang, N., Antal, A., et al. (2008). Transcranial direct current stimulation: state of the art 2008. Brain Stimul. 1, 206-223. doi: 10.1016/j.brs.2008. 06.004

Nitsche, M. A., Liebetanz, D., Lang, N., Antal, A., Tergau, F., and Paulus, W. (2003a). Safety criteria for transcranial direct current stimulation (tDCS) in humans. Clin. Neurophysiol. 114, 2220-2222; author reply 2222-2223. doi: 10.1016/S13882457(03)00235-9

Nitsche, M. A., Schauenburg, A., Lang, N., Liebetanz, D., Exner, C., Paulus, W., etal. (2003b). Facilitation of implicit motor learning by weak transcranial direct current stimulation of the primary motor cortex in the human. J. Cogn. Neurosci. 15, 619-626. doi: 10.1162/089892903321662994

Nitsche, M. A., and Paulus, W. (2000). Excitability changes induced in the human motor cortex by weak 
transcranial direct current stimulation. J. Physiol. 527(Pt 3), 633-639. doi: 10.1111/j.1469-7793.2000.t01-100633.x

Nitsche, M. A., and Paulus, W. (2001). Sustained excitability elevations induced by transcranial DC motor cortex stimulation in humans. Neurology 57, 1899-1901. doi: 10.1212/WNL.57.10.1899

Nyberg, L., Persson, J., Habib, R., Tulving, E., McIntosh, A. R., Cabeza, R., et al. (2000). Large scale neurocognitive networks underlying episodic memory. J. Cogn. Neurosci. 12, 163 173. doi: 10.1162/089892900561805

Park, D. C., Polk, T. A., Park, R., Minear, M., Savage, A., and Smith, M. R. (2004). Aging reduces neural specialization in ventral visual cortex. Proc. Natl. Acad. Sci. U.S.A. 101, 13091-13095. doi: 10.1073/ pnas.0405148101

Park, D. C., and Reuter-Lorenz, P. (2009). The adaptive brain: aging and neurocognitive scaffolding. Annu. Rev. Psychol. 60, 173-196. doi: 10.1146/annurev.psych.59.103006. 093656

Penolazzi, B., Di Domenico, A., Marzoli, D., Mammarella, N., Fairfield, B., Franciotti, R., et al. (2010). Effects of transcranial direct current stimulation on episodic memory related to emotional visual stimuli. PLoS ONE 5:e10623. doi: 10.1371/journal.pone.0010623

Poldrack, R. A., Wagner, A. D., Prull, M. W., Desmond, J. E., Glover, G. H., and Gabrieli, J. D. (1999). Functional specialization for semantic and phonological processing in the left inferior prefrontal cortex. Neuroimage 10, 15-35. doi: 10.1006/nimg. 1999.0441
Poreisz, C., Boros, K., Antal, A., and Paulus, W. (2007). Safety aspects of transcranial direct current stimulation concerning healthy subjects and patients. Brain Res. Bull. 72, 208-214. doi: 10.1016/ j.brainresbull.2007.01.004

Priori, A. (2003). Brain polarization in humans: a reappraisal of an old tool for prolonged non-invasive modulation of brain excitability. Clin. Neurophysiol. 114, 589595. doi: 10.1016/S1388-2457(02) 00437-6

Rodriguez-Ferreiro, J., Gennari, S. P., Davies, R., and Cuetos, F. (2011). Neural correlates of abstract verb processing. J. Cogn. Neurosci. 23, 106-118. doi: 10.1162/ jocn.2010.21414

Rugg, M. D., and Wilding, E. L. (2000). Retrieval processing and episodic memory. Trends Cogn. Sci. $4, \quad 108-115$. doi: 10.1016/S1364 6613(00)01445-5

Sabsevitz, D. S., Medler, D. A., Seidenberg, M., and Binder, J. R. (2005). Modulation of the semantic system by word imageability. Neuroimage 27, 188-200. doi: 10.1016/j.neuroimage.2005.04.012

Schacter, D. L., Savage, C. R., Alpert, N. M., Rauch, S. L., and Albert, M. S. (1996). The role of hippocampus and frontal cortex in age-related memory changes: a PET study. Neuroreport 7, 1165-1169. doi: 10.1097/00001756199604260-00014

Simons, J. S., and Spiers, H. J. (2003). Prefrontal and medial temporal lobe interactions in long-term memory. Nat. Rev. Neurosci. 4, 637-648. doi: 10.1038/nrn1178

Spaniol, J., Davidson, P. S., Kim, A. S., Han, H., Moscovitch, M., and
Grady, C. L. (2009). Event-related fMRI studies of episodic encoding and retrieval: meta-analyses using activation likelihood estimation. Neuropsychologia 47, 1765-1779. doi: 10.1016/j.neuropsychologia.2009. 02.028

Spencer, W. D., and Raz, N. (1995). Differential effects of aging on memory for content and context: a meta-analysis. Psychol. Aging 10 527-539. doi: 10.1037/0882-7974.10. 4.527

Thickbroom, G. W. (2007). Transcranial magnetic stimulation and synaptic plasticity: experimental framework and human models. Exp. Brain Res. 180, 583-593. doi: 10.1007/s00221007-0991-3

Tulving, E. (1983). Elements of Episodic Memory. London: Oxford University Press.

Tulving, E., Kapur, S., Craik, F. I., Moscovitch, M., and Houle, S. (1994). Hemispheric encoding/retrieval asymmetry in episodic memory: positron emission tomography findings. Proc. Natl. Acad. Sci. U.S.A. 91, 1989-1991. doi: 10.1073/pnas.91.6.2016

Wagner, A. D., Poldrack, R. A., Eldridge, L. L., Desmond, J. E., Glover, G. H., and Gabrieli, J. D. (1998). Materialspecific lateralization of prefrontal activation during episodic encoding and retrieval. Neuroreport 9, 3711-3717. doi: 10.1097/00001756 199811160-00026

Ziemann, U., and Siebner, H. R. (2008). Modifying motor learning through gating and homeostatic metaplasticity. Brain Stimul. 1, 60-66. doi: 10.1016/j.brs.2007.08.003

Zimerman, M., Nitsch, M., Giraux, P., Gerloff, C., Cohen, L. G., and Hummel, F. C. (2013). Neuroenhancement of the aging brain restoring skill acquisition in old subjects. Ann. Neurol. 73, 10-15. doi: 10.1002/ana.23761

Zollig, J., and Eschen, A. (2009). Measuring compensation and its plasticity across the lifespan. Restor. Neurol. Neurosci. 27, 421-433. doi: 10.3233/RNN-2009-0513

Conflict of Interest Statement: The authors declare that the research was conducted in the absence of any commercial or financial relationships that could be construed as a potential conflict of interest.

Received: 01 August 2013; paper pending published: 09 August 2013; accepted: 22 August 2013; published online: 11 September 2013.

Citation: Manenti $R$, Brambilla $M$, Petesi $M$, Ferrari $C$ and Cotelli $M$ (2013) Enhancing verbal episodic memory in older and young subjects after non-invasive brain stimulation. Front. Aging Neurosci. 5:49. doi: 10.3389/fnagi.2013.00049

This article was submitted to the journal Frontiers in Aging Neuroscience.

Copyright (C) 2013 Manenti, Brambilla, Petesi, Ferrari and Cotelli. This is an open-access article distributed under the terms of the Creative Commons Attribution License (CC BY). The use, distribution or reproduction in other forums is permitted, provided the original author(s) or licensor are credited and that the original publication in this journal is cited, in accordance with accepted academic practice. No use, distribution or reproduction is permitted which does not comply with these terms. 